\title{
Advanced optics in an interdisciplinary graduate program
}

\section{S. Nic Chormaic}

S. Nic Chormaic, "Advanced optics in an interdisciplinary graduate program," Proc. SPIE 9289, 12th Education and Training in Optics and Photonics Conference, 92890M (17 July 2014); doi: 10.1117/12.2070380

SPIE Event: 12th Education and Training in Optics and Photonics Conference, 2013, Porto, Portugal 


\title{
Advanced optics in an interdisciplinary graduate program
}

\author{
S. Nic Chormaic* \\ Light-Matter Interactions Unit, OIST Graduate University, 1919-1 Tancha, Onna-son, Okinawa 904- \\ 0495, Japan
}

\begin{abstract}
The Okinawa Institute of Science and Technology Graduate University, established in November 2011, provides a 5year interdisciplinary $\mathrm{PhD}$ program, through English, within Japan. International and Japanese students entering the program undertake coursework and laboratory rotations across a range of topics, including neuroscience, molecular science, physics, chemistry, marine science and mathematics, regardless of previous educational background. To facilitate interdisciplinarity, the university has no departments, ensuring seamless interactions between researchers from all sectors. As part of the $\mathrm{PhD}$ program a course in Advanced Optics has been developed to provide $\mathrm{PhD}$ students with the practical and theoretical skills to enable them to use optics tools in any research environment. The theoretical aspect of the course introduces students to procedures for complex beam generation (e.g. Laguerre-Gaussian), optical trapping, beam analysis and photon optics, and is supported through a practical program covering introductory interference/diffraction experiments through to more applied fiber optics. It is hoped that, through early exposure to optics handling and measurement techniques, students will be able to develop and utilize optics tools regardless of research field. In addition to the formal course in Advanced Optics, a selection of students also undertakes 13 week laboratory rotations in the Light-Matter Interactions research laboratory, where they work side-by-side with physicists in developing optics tools for laser cooling, photonics or bio-applications. While currently in the first year, conclusive results about the success of such an interdisciplinary $\mathrm{PhD}$ training are speculative. However, initial observations indicate a rich cross-fertilization of ideas stemming from the diverse backgrounds of all participants.
\end{abstract}

Keywords: Structured $\mathrm{PhD}$, interdisciplinarity, optics training

\section{INTRODUCTION}

The Okinawa Institute of Science and Technology (OIST) Graduate University (www.oist.jp), based on the sub-tropical island of Okinawa, Japan was formally inaugurated in November 2011, with the first student intake to the 5-year interdisciplinary $\mathrm{PhD}$ in Science program in September 2012. With more than half of the University faculty and graduate students recruited from outside Japan, English is the language of instruction and operation at all levels. Currently, there are five main research areas at OIST, covering the Physical Sciences (physics and chemistry, with a stronger focus on physics), Neuroscience, Molecular Sciences, Environmental and Ecological Sciences, and Mathematical Computational Sciences. Prior to accreditation as a Graduate University in 2011, OIST was recognized for leading research in several of these fields, with a particular emphasis on the Life Sciences.

The establishment of a graduate university on Okinawa was initially discussed in 2001 and four Japanese Initial Research Projects (IRP) commenced in 2004 as a foundation upon which the university was later built. The formation of the Okinawa Institute of Science and Technology Promotion Corporation (PC) was approved by the Diet (Japanese Government) in 2005 to prepare for the realization of the graduate university in 2011. Aside from a strong research basis stemming from the IRPs and the PC, OIST also had a reputation by then for hosting international conferences and shortterm graduate level training courses. Based on a firm foundation in the basic sciences, OIST Graduate University promotes education that is highly interdisciplinary. The 5-year graduate program features interactive teaching with tutorial-style courses providing preparation for thesis research. Program design is customized to the unique needs of individual students, based on their educational and career backgrounds. The ethos of the University is that, from the beginning, students work side-by-side with faculty and researchers in well-equipped laboratories, learning research by doing research.

*sile.nicchormaic@oist.jp; www.oist.jp

12th Education and Training in Optics and Photonics Conference, edited by

Manuel F. P. C. Martins Costa, Mourad Zghal, Proc. of SPIE Vol. 9289, 92890M

(c) 2014 SPIE, OSA, IEEE, ICO · doi: 10.1117/12.2070380

Proc. of SPIE Vol. $928992890 \mathrm{M}-1$ 
With 45 faculty members (40 full-time and 5 adjuncts, faculty expansion phases in targeted areas are under way) and a student population of only 35 in the first year, OIST has created a unique environment for student supervision and guidance. The aim is to average a ratio of 2 to 1 for student to faculty over the 5 -year $\mathrm{PhD}$ cycle with approximately 20 new students accepted onto the program each year, with the number being admitted determined by the standard of applicants. While students enter with a prior education in a core discipline, the structure of the PhD program is such that, while they extend their knowledge in their core discipline, they are also exposed to other disciplines through elective course work and mandatory laboratory rotations in a way to promote cross-disciplinary research and fertilization.

\section{PHD PROGRAM}

OIST Graduate University only offers a 5-year PhD program to entrants; there are no BSc or MSc degrees awarded. The first batch of students entered in September 2012, representing 18 different nationalities out of the 35 entrants. These students had core disciplines spread across biological sciences, chemistry, computing, mathematics, neuroscience, physics, and engineering. The nationality and prior background distribution of students entering the 2012 program is shown in Figure 1. Note that in relation to the gender of those admitted, $25 \%$ are female, and they are relatively evenly distributed across all disciplines. With 18 nationalities to educate in such a small group, challenges related to culture, language, student expectations, and student preparation for a $\mathrm{PhD}$ program are being faced every day. Regardless of background, all students entered the same program with some individualization offered. For example, while the base program is 5-years long, it is envisioned that students entering with an MSc qualification may take less time to complete compared to students entering with a BSc degree, depending on the relevancy of the MSc training. The language used for both instruction and research in the University is English. Students are offered language training in English and/or Japanese depending on their needs.
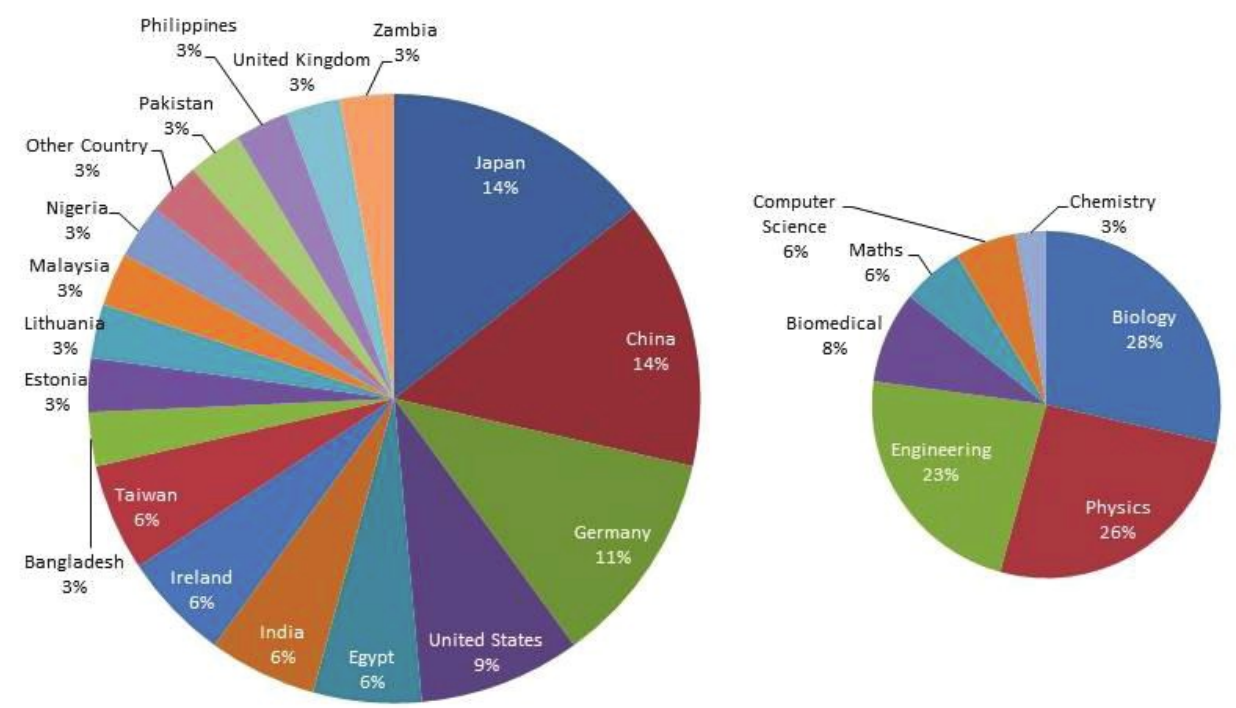

Figure 1. Student distribution for the 2012 intake to the OIST PhD Program. Left: Nationality distribution; Right: Previous area of study distribution. The total number of students is 35 .

During the first year, all students undertake three laboratory rotations in research groups at the University, in addition to following advanced courses in their core area, or more introductory courses outside their core area, to encourage the inter- and cross-disciplinarity of the program. Each research rotation takes 13 weeks and can see students having to undertake research in an area with which they are completely unfamiliar, e.g. a student with a background in biology, having no third level physics education, may have to do a rotation project in a quantum physics theory group. While rotation projects are designed to suit the student entering the research unit, it is also essential that they push the student beyond their comfort zone and force them to apply the techniques that they have acquired from their core discipline to an entirely new problem or to learn new methods that they can later use in their own research work. 
In addition to the rotations, students have to attend a certain number of taught courses during the first and second year of the program for credit accumulation. About 40 different courses are on offer, most of which do not have prerequisites and are designed to deal with students at all different knowledge levels in the classroom. This is in stark contrast to many graduate programs and brings major challenges from a pedagogical point of view. While keeping a student with a background in the topic interested - and challenged - to graduate level, how can one also engage a student who is crossing a boundary and has no prior background in the topic or in related topics? Keeping in mind the very low student numbers at the University, this also crosses into the classroom where class sizes vary from one single student to a maximum of about 12 students in any given course. Since the program is in its infancy, it is expected that course arrangement and student evaluation will evolve based on experiences during the initial years. Current practice is to evaluate performance based on international standards and/or on student background on entering and progress made.

In the Second Year of the program, students identify a research laboratory for a 1-year research project and complete any course work that is needed to achieve the required credits for progressing to the $\mathrm{PhD}$ research project. It is assumed that most students will complete their $\mathrm{PhD}$ within the research group where this 1-year project is conducted. At the end of the Second Year, the student must write a $\mathrm{PhD}$ project proposal and defend it before being accepted to and embarking on the pure research aspect of their degree. We have not yet reached this stage of the procedure with our initial batch of intake students and, therefore, cannot yet determine if any students finally choose to study outside their core discipline on entry to the program. Naturally, the success of the interdisciplinary training during the first two years will be judged on the basis of whether any students do veer away from their original area significantly, or whether they even succeed in integrating techniques from other disciplines successfully into their PhD projects. It is important to note that the lack of a formal structure within the university, i.e. no departments, schools, or faculties, does significantly increase interaction between $\mathrm{PhD}$ students of all disciplines within the University and this should certainly impact on ideas spreading between different areas and the adaptation of techniques for new areas. All students live in campus accommodation during (at least) the first year and interactions are very strong, with no evidence of a discipline-based divide amongst them.

\section{ADVANCED OPTICS AT OIST GRADUATE UNIVERSITY}

The Advanced Optics course at OIST is designed to expose PhD students in the First or Second Year of the 5-year program to optics, with the aim of enabling them to apply optics-based techniques and design in laboratories, regardless of discipline. The course consists of traditional lectures, hands-on laboratory work, and journal club presentations and critiques, related, where appropriate, to the student's discipline. The distribution of students for the 2012 academic year (the first year of the program) is shown in Figure 2. Since OIST aims to have small numbers in each course for individualized $\mathrm{PhD}$ program development the number of students taking the module was about average for courses at OIST and totaled five, all of whom are male. All students enrolled on the course had a reasonable level of mathematical ability and $60 \%$ had prior exposure to optics. While this can be viewed as a challenge, the fact that all had a reasonable mathematical ability having entered through the physical sciences ensured that the course was maintained at graduate level, without the need to redress some deficits in this regard. Naturally, some students had to cover additional background material related to optics as self-study.

The coursework covers wave and quantum properties of light, geometrical optics, wave optics including Fresnel and Fraunhofer diffraction, interaction of photons and atoms, and applications of optics such as fiber optics (multimode and single-mode), laser resonators, optical tweezing and trapping, and non-linear optics. Relevance to current research in optics-based fields is emphasized by incorporating research laboratory visits that tie in with the delivered material.

The current laboratory sessions consist of teaching modules in interferometry, diffraction, waveguides, and optical fibers, from a very introductory level to more advanced. It is planned to integrate further experimental modules in the future, including saturated absorption spectroscopy of rubidium, the excitation of higher order modes using a spatial light modulator, and photon entanglement generation, to cater for students entering with a high level of undergraduate optics.

It is also essential to provide the students with some training in the handling of delicate optics, maintenance of a laboratory notebook, errors in measurement, etc. The time allotted to the course was not sufficient to provide an indepth treatment of these auxiliary topics, but in future years the first week of laboratory will be dedicated to such general skills training rather than commencing immediately with the experimental program. 
A clear difference in the prior exposure to laboratory skills training was evident, despite the small number of students in the class. This was addressed in the laboratory sessions, but could become a real challenge if students from outside the physical sciences were to attend this course. While the title "Advanced Optics" may be off-putting to some students without prior optics background, other students may desire the challenge it offers them. A pedagogical approach to the teaching of students with different backgrounds needs to be maintained, to avoid the risk of alienating some students within the classroom by over or under challenging them.
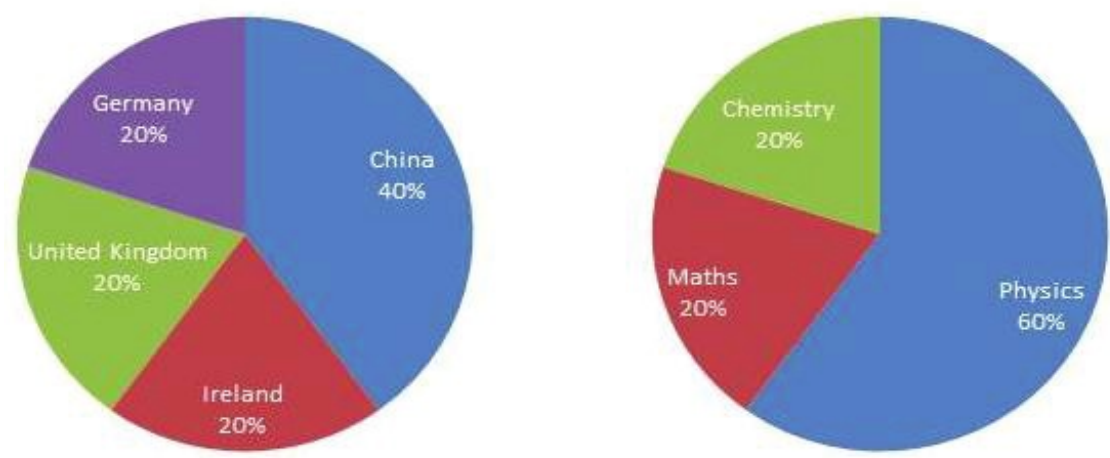

Figure 2. Student distribution for the 2012 intake to the Advanced Optics Module of the OIST PhD Program. Left: Nationality distribution; Right: Previous area of study distribution. The total number of students is 5.

Aside from the Advanced Optics laboratory and coursework, some students are assigned to undertake optics-based, 13week laboratory rotations within the Light-Matter Interactions Unit (groups.oist.jp/light). To date, one physics student has successfully completed a rotation research project on nanomachining of optical nanofibers using focused ion beam milling. In stark contrast, biology students assigned to rotations in this (clearly) physics laboratory have chosen to not complete the rotation project. This is in spite of the projects being designed with their background in mind to minimize the culture shock of entering a laser physics laboratory for students with no physics since high school. This indicates some of the challenges faced by faculty at OIST - life sciences students frequently have a dislike or disinterest in physics that is hard to overcome and it is often due to their perception of the "difficulty" associated with physics and the mathematical ability required to be a physicist. If we manage to break down this barrier and expose life sciences students to physics research, whether it is in basic theoretical modeling or laboratory experiments, the skillset they develop (programming, instrumentation, data analysis, project planning, resource management) should hold them in good stead throughout their chosen careers.

\section{CONCLUSION}

While the OIST Graduate Program is still in its infancy, it has been designed to expose PhD students to a range of courses and laboratory research experiences during the first two years of their $\mathrm{PhD}$ program in a structured manner and strives to internationalize Japanese graduate education. Selection to the program is highly competitive, resulting in a student body that is very international and covers a range of background disciplines in the sciences and engineering. Students are not admitted to a specific discipline and OIST has challenged traditional university structures by avoiding the formation of departments, schools and faculties. Based on student participation in rotations and the Advanced Optics course, a general Laboratory Skills Training course would be very beneficial to all the students and could be delivered as a cross-disciplinary short course over a 1-2 day period to all students entering OIST. Surprisingly, most students had never had to maintain a laboratory-specific notebook during their undergraduate studies. While this is understandable for students with a theoretical background, it is less obvious why this is the case for those with an experimental background and showed clear deficits in skills training that many science undergraduates receive. In the optics laboratory, while some students had experience on paper as evidenced by their transcripts, in reality most had never had to set up an optical bench or consider the arrangement of components in an optical setup in order to demonstrate even the most basic of optics effects such as interference and diffraction. With such a visual subject matter as optics, it is surprising that more universities do not focus student education on understanding through seeing, rather than learning by rote. We hope to overcome some of these deficits by training and educating students across a range of disciplines and exposing them not only to topics outside their area of choice, but also to an international environment that should help them develop as better scientists in the long term. 